\title{
High expression of $B C L 6$ inhibits the differentiation and development of hematopoietic stem cells and affects the growth and development of chickens
}

Hongmei $\mathrm{Li}^{1,2,3+}$, Bowen Hu ${ }^{1,2,3 \dagger}$, Shang Hu$u^{1,2,3}$, Wen Luo ${ }^{1,2,3}$, Donglei Sun ${ }^{4}$, Minmin Yang ${ }^{1,2,3}$, Zhiying Liao ${ }^{1,2,3}$, Haohui Wei ${ }^{1,2,3}$, Changbin Zhao ${ }^{1,2,3}$, Dajian Li ${ }^{1,2,3}$, Meiqing Shi ${ }^{4}$, Qingbin Luo ${ }^{1,2,3}$, Dexiang Zhang ${ }^{1,2,3}$,

Qinghua Nie ${ }^{1,2,3}$ and Xiquan Zhang ${ }^{1,2,3^{*}}$ (D)

\begin{abstract}
Background: B-cell CLL/lymphoma 6 (BCL6) is a transcriptional master regulator that represses more than 1200 potential target genes. Our previous study showed that a decline in blood production in runting and stunting syndrome (RSS) affected sex-linked dwarf (SLD) chickens compared to SLD chickens. However, the association between BCL6 gene and hematopoietic function remains unknown in chickens.

Methods: In this study, we used RSS affected SLD (RSS-SLD) chickens, SLD chickens and normal chickens as research object and overexpression of BCL6 in hematopoietic stem cells (HSCs), to investigate the effect of the BCL6 on differentiation and development of HSCs.

\footnotetext{
* Correspondence: xqzhang@scau.edu.cn

${ }^{\dagger}$ Hongmei Li and Bowen Hu contributed equally to this work.

'Department of Animal Genetics, Breeding and Reproduction, College of Animal Science, South China Agricultural University, Guangzhou 510642, Guangdong Province, China

${ }^{2}$ Guangdong Provincial Key Lab of AgroAnimal Genomics and Molecular Breeding and Key Lab of Chicken Genetics, Breeding and Reproduction, Ministry of Agriculture, Guangzhou 510642, Guangdong, China

Full list of author information is available at the end of the article
}

C The Author(s). 2021 Open Access This article is licensed under a Creative Commons Attribution 4.0 International License, which permits use, sharing, adaptation, distribution and reproduction in any medium or format, as long as you give appropriate credit to the original author(s) and the source, provide a link to the Creative Commons licence, and indicate if changes were made. The images or other third party material in this article are included in the article's Creative Commons licence, unless indicated otherwise in a credit line to the material. If material is not included in the article's Creative Commons licence and your intended use is not permitted by statutory regulation or exceeds the permitted use, you will need to obtain permission directly from the copyright holder. To view a copy of this licence, visit http://creativecommons.org/licenses/by/4.0/. The Creative Commons Public Domain Dedication waiver (http://creativecommons.org/publicdomain/zero/1.0/) applies to the data made available in this article, unless otherwise stated in a credit line to the data. 
(Continued from previous page)

Results: The results showed that comparison of RSS-SLD chickens with SLD chickens, the BCL6 was highly expressed in RSS-SLD chickens bone marrow. The bone marrow of RSS-SLD chickens was exhausted and red bone marrow was largely replaced by yellow bone marrow, bone density was reduced, and the levels of immature erythrocytes in peripheral blood were increased. At the same time, the hematopoietic function of HSCs decreased in RSS-SLD chickens, which was manifested by a decrease in the hematopoietic growth factors (HGFs) EPO, SCF, TPO, and IL-3, as well as hemoglobin $a_{1}$ and hemoglobin $\beta$ expression. Moreover, mitochondrial function in the HSC of RSS-SLD chickens was damaged, including an increase in ROS production, decrease in ATP concentration, and decrease in mitochondrial membrane potential $\left(\Delta \psi_{\mathrm{m}}\right)$. The same results were also observed in SLD chickens compared with normal chickens; however, the symptoms were more serious in RSS-SLD chickens. Additionally, after overexpression of the BCL6 in primary HSCS, the secretion of HGFs (EPO, SCF, TPO and IL-3) was inhibited and the expression of hemoglobin $a_{1}$ and hemoglobin $\beta$ was decreased. However, cell proliferation was accelerated, apoptosis was inhibited, and the HSCs entered a cancerous state. The function of mitochondria was also abnormal, ROS production was decreased, and ATP concentration and $\Delta \psi_{\mathrm{m}}$ were increased, which was related to the inhibition of apoptosis of stem cells.

Conclusions: Taken together, we conclude that the high expression of BCL6 inhibits the differentiation and development of HSCs by affecting mitochondrial function, resulting in impaired growth and development of chickens. Moreover, the abnormal expression of BCL6 might be a cause of the clinical manifestations of chicken comb, pale skin, stunted growth and development, and the tendency to appear RSS in SLD chickens.

Keywords: BCL6, Hematopoietic stem cells, Mitochondrial function, Runting and stunting syndrome, Sex-linked dwarf chickens

\section{Background}

B-cell CLL/lymphoma 6 (BCL6) is known as a carcinogenic driver. The protein of BCL6 has two functional domains, including the hydrophobic $\mathrm{BTB} / \mathrm{POZ}$ region at the $\mathrm{N}$-terminal and the functional domain of zinc-finger structures that have homology with members of the Cterminal Kruppel subfamily of zinc-finger proteins [1]. BCL6 specifically binds to the promoter DNA of the target gene by the zinc-finger domain, thereby inhibiting the transcription of target genes. At present, research on BCL6 mainly focuses on the structure formation of the B-cell germinal center, because BCL6 is also closely related to diffuse large B-cell lymphoma (germinal center type), and is thought to be a target for the treatment of cancer and autoimmune diseases.

Hematopoietic stem cells (HSCs) are primitive cells with the potential for self-renewal and multipolarity. Most HSCs are dormant, and only a small number can self-renew and differentiate into other types of daughter cells, which are important for maintaining the stability of the HSC bank and blood system in vivo [2]. Thus, under normal circumstances, most HSCs are in the nonproliferative $G_{0}$ phase [3]. When HSCs and early hematopoietic progenitor cells are stimulated or positively regulated, or when the balance between the stimulus and the cytokine is disturbed, HSCs begin to proliferate [4].

In vertebrates, most blood production occurs in the bone marrow. HSCs exist in specific microenvironmental niches in the bone marrow. Bone marrow mesenchymal stem cells (MSCs) can support HSCs and provide various hematopoietic cytokines, which build a good spatial environment for the proliferation, differentiation, and maturation of HSCs. Hemopoietic growth factors (HGFs) mainly bind to related receptors, and transmit growth and differentiation signals, to regulate the survival and proliferation of hematopoietic stem/progenitor cells and to stimulate their differentiation, maturation, and release into various blood cells [5]. Previous studies have shown that bone marrow MSCs secrete various hematopoietic cytokines, including FIT3 ligands, TPO, SCF, G-CSF, GMCSF, IL-3, IL-6, and LIF [6].

Moreover, most dormant HSCs are exposed to low oxygen levels, which may inhibit the proliferation of HSCs and maintain the dormant state of HSCs. In fact, even HSCs in the peripheral circulation show a hypoxia spectrum [7]. When HSCs are in a hypoxic state during the dormant period, low metabolic levels are maintained mainly by cytoplasmic glycolysis. Once the HSCs are activated into the proliferation and differentiation stage, they need the mitochondrial tricarboxylic acid cycle to produce large amounts of energy, because at this time the consumption of oxygen greatly increases [8]. Therefore, the energy metabolism of HSCs can be analyzed to predict their cell state. To date, the association between the BCL6 gene and hematopoietic function still remains unknown in chickens.

Sex-linked dwarf (SLD) chickens are caused by mutations or deletions in the GHR gene, which are small in size (only $60-70 \%$ of normal chicken weight). They are 
particularly prone to appear runting and stunting syndrome (RSS) [9], which is characterized by low body weight, generally occurs early in life, and leads to considerable economic losses in the commercial broiler industry [10]. In a previous study, we observed a decline in blood production in RSS affected SLD (RSS-SLD) chickens compared to SLD chickens [9]. In this study, we used RSS-SLD chickens, SLD chickens and normal chickens as research object and overexpression of the BCL6 in HSCs, to study the effect of the BCL6 on differentiation and development of chicken HSCs.

\section{Methods}

\section{Ethics statement}

All animal experiments were performed according to the protocols approved by the South China Agriculture University Institutional Animal Care and Use Committee (approval number SCAU\#0017). All animal procedures followed the regulations and guidelines established by this committee and minimized the suffering of animals.

\section{Chickens}

The 140-day-old Cantonese-yellow chickens used in the living experiment were provided by the breeding farm of South China Agricultural University. The 14-day-old chickens used for hematopoietic stem cell isolation were provided by Zhejiang GD poultry Co., Ltd.

In all, 6 RSS affected SLD (RSS-SLD) chickens, 6 SLD chickens and 6 normal chickens at 140-day-old of age in strain Cantonese-yellow chickens were utilized to explore the molecular mechanism of the BCL6 in vivo by regulating the differentiation and development of HSCs to affect the growth characteristics of chicken as previously described [9]. The 14-day-old normal chickens were only utilized for HSCs and MSCs isolation to study the function of the BCL6 in vitro.

\section{Paraffin sections and HE staining}

The samples of 140-day-old RSS-SLD chickens, SLD chickens and normal chickens were fixed with $10 \%$ neutral formalin for 5 days and then immersed in hydrochloric acid/formic acid working solution to complete the decalcification. After decalcification, the samples were dehydrated in alcohol and transformed into a transparent state using xylene. After the transparency step was completed, the samples were soaked in wax and embedded in paraffin. A paraffin sectioning machine was used to cut $7-10 \mu \mathrm{m}$ sections, which were stained with hematoxylin and eosin.

\section{Red blood cell count}

Blood samples of 140-day-old RSS-SLD chickens, SLD chickens and normal chickens were diluted, and the number of red blood cells was calculated using a blood cell count board.

\section{Hematocrit determination}

Blood samples of 140-day-old RSS-SLD chickens, SLD chickens and normal chickens were collected into tubes containing EDTA, which was drawn into a capillary tube that was sealed with glue at the bottom. Each sample was centrifuged at $3000 \mathrm{r} / \mathrm{min}$ for $30 \mathrm{~min}$, the blood is divided into three layers. The ratio of the bottom red blood cell layer to the whole cell column was calculated and taken as the level of hematocrit.

\section{Blood smear and Wright-Giemsa staining}

Each blood sample of 140-day-old RSS-SLD chickens, SLD chickens and normal chickens was uniformly coated on a slide and allowed to dry naturally. Then two to three drops of Wright-Giemsa dye were added to completely cover the specimen and the sample was subjected to staining for 1-2 min. Next, the same amount of phosphate buffer as the dye ( $\mathrm{pH}$ 6.4) was added, the sample was shaken slowly, and the staining was allowed to proceed for another 3-5 min. The whole process must keep the sample moist to prevent pigment deposition. Finally, the sample was washed, dried, and inspected under an optical microscope (Leica, Germany).

\section{Quantitative real-time PCR}

RNA was extracted from tissues or cells using RNAiso reagent (Takara, Japan) according to the manufacturer's protocol. The concentration of RNA samples and OD value of 260/280 were detected using a Nanodrop 2000c spectrophotometer (Thermo, USA). Samples were stored at $-80^{\circ} \mathrm{C}$ for later use. cDNA was synthesized using a PrimeScript RT Reagent Kit (Takara, Japan) for quantitative real-time PCR (qRT-PCR). The MonAmp ${ }^{\text {no }}$ ChemoHS qPCR Mix (Monad Co., LTD, Guangzhou, China) was used for qRT-PCR using a Bio-Rad CFX96 Real-Time Detection instrument (Bio-Rad, USA) according to the manufacturer's protocol. The reaction procedure included initial denaturation at $95^{\circ} \mathrm{C}$ for $3 \mathrm{~min}$, followed by denaturation at $95^{\circ} \mathrm{C}$ for $10 \mathrm{~s}$, annealing at $60^{\circ} \mathrm{C}$ for $20 \mathrm{~s}$, extension at $72{ }^{\circ} \mathrm{C}$ for $10 \mathrm{~s}$, for a total of 40 cycles. At the end of the cycle, the dissolution curve was analyzed and the detection temperature was $70-95^{\circ} \mathrm{C}$. Relative gene expression was measured using $\mathrm{qRT}$-PCR twice for each reaction, and GADPH was used as a control. The primers used for qRT-PCR are listed in Table 1.

\section{Extraction of peripheral blood HSCs, bone marrow HSCs, and MSCs}

Peripheral blood HSCs, bone marrow HSCs, and MSCs were extracted using the appropriate separation kits 
Table 1 Primer sequences of qRT-PCR

\begin{tabular}{|c|c|c|c|}
\hline Genes & Primer sequences $\left(5^{\prime} \rightarrow 3^{\prime}\right)$ & Temperature, ${ }^{\circ} \mathrm{C}$ & Size, bp \\
\hline \multirow[t]{2}{*}{$B C L 6$} & GCAGTTCAGAGCCCACAAAA & 58 & 205 \\
\hline & GTTCAGACGGGAGGTGTACA & & \\
\hline \multirow[t]{2}{*}{ Hemoglobin $a_{1}$} & GTCAACTTCAAACTCCTGGGC & 57 & 140 \\
\hline & TAACGGTACTTGGCGGTCAG & & \\
\hline \multirow[t]{2}{*}{ Hemoglobin $\beta$} & AGAACTTCAGGCTCCTGGGTG & 57 & 167 \\
\hline & GTGCTCCGTGATCTITGGTG & & \\
\hline \multirow[t]{2}{*}{ GAPDH } & TCCTCCACCTTTGATGCG & $50-65$ & 225 \\
\hline & GTGCCTGGCTCACTCCTT & & \\
\hline
\end{tabular}

(TBDscience, China) following the manufacturer's protocol.

\section{Cell culture}

HSCs and MSCs were cultured in Iscove's Modified Dulbecco's Medium (Gibco, USA) with 15\% fetal bovine serum (ExCell Bio, China) and $0.2 \%$ penicillin/streptomycin (Invitrogen, USA). All cells were cultured at $37^{\circ} \mathrm{C}$ in a $5 \% \mathrm{CO}_{2}$ humidified atmosphere.

\section{Transfection}

Cells were plated onto a culture plate and incubated overnight prior to the transfection experiment. Transfection was performed using Lipofectamine 3000 reagent (Invitrogen, USA) following the manufacturer's protocol, and nucleic acids were diluted in OPTI-MEM Medium (Gibco, USA). All cells were analyzed $48 \mathrm{~h}$ after transfection.

\section{Plasmid construction}

The plasmid pcDNA3.1-BCL6 was generated by amplifying $B C L 6$ coding sequences from RNA by RT-PCR, and then subsequently cloning them into the pcDNA3.1 vector (Promega, USA) using a pMD18-T cloning vector (Takara, China) with the EcoRI and HindIII restriction sites. Plasmid constructs were confirmed by Sanger sequencing. The primers utilized for vector construction were showed in Table 2 and synthesized by Sangon Biotech (Shanghai, China).

\section{Detection of reactive oxygen species}

The production of reactive oxygen species (ROS) in the mitochondria of cells was measured using an ROS assay kit (Beyotime, China) according to the manufacturer's protocol. Dichlorofluorescein (DCF) fluorescence was

Table 2 Primer amplification of target gene BCL6 CDS region

\begin{tabular}{lll}
\hline Primers & Primer sequences $\left(\mathbf{5}^{\prime} \rightarrow \mathbf{3}^{\prime}\right)$ & Size, bp \\
\hline BCL6-CDS-F & CCGGAATTCATGGCCTCACCGGCAGACAGCTGC & 2127 \\
BCL6-CDS-R & CCCAAGCTTTCAGCAAGCCTTGGGGAGCTC & \\
\hline
\end{tabular}

determined using a Fluorescence/Multi-Detection Microplate Reader (BioTek, USA) as previously described [11]. Data were normalized to the control group and are expressed as a percentage of control levels.

\section{Detection of ATP content}

ATP levels were measured using an ATP assay kit (Beyotime, China) according to the manufacturer's protocol. A Fluorescence/Multi-Detection Microplate Reader (BioTek, USA) was used to determine ATP levels. Data were normalized to the control group and are expressed as a percentage of control levels.

\section{Detection of mitochondrial membrane potential}

Mitochondrial membrane potential $(\Delta \Psi \mathrm{m})$ was measured using a JC-1 kit (Beyotime, China) according to the manufacturer's protocol. The mitochondria were fixed with JC-1. The fluorescence was determined using a Fluorescence/Multi-Detection Microplate Reader (BioTek, USA) after the cells were incubated with JC-1 for $20 \mathrm{~min}$ at $37^{\circ} \mathrm{C} ; 10 \mu \mathrm{mol} / \mathrm{L}$ rotenone was used as a standard inhibitor of $\Delta \Psi \mathrm{m}$. The data (the ratio of aggregated and monomeric JC-1) were normalized to the control group and are expressed as a percentage of control levels.

\section{Cytokine levels determined by ELISA}

ELISA was used to detect the content of various HGFs in vivo and in vitro according to the instructions of commercial ELISA kits (mlbio, China). In brief, the plasma of 140-day-old chickens with EDTA and cell culture supernatant were centrifuged at $4000 \times g$ for $5 \mathrm{~min}$ at $4{ }^{\circ} \mathrm{C}$. Then, the supernatant of $10 \mu \mathrm{L}$ samples was collected and added to the bottom of the plate well with $40 \mu \mathrm{L}$ sample diluents along with $100 \mu \mathrm{L}$ enzyme-labeled reagents. After the plate was sealed with the sealing film, the plate was incubated at $37^{\circ} \mathrm{C}$ for $60 \mathrm{~min}$. Finally, we performed washing, color rendering and termination of the plate following standard procedures. Absorbance at $450 \mathrm{~nm}$ was determined using a Fluorescence/Multi-Detection Microplate Reader (BioTek, USA). 


\section{Statistical analyses}

All experiments were performed at least three times. The data are presented as means \pm standard error of the mean (SEM). Statistical analyses were performed using Student's $t$-test, and we considered $P<0.05$ to be statistically significant, ${ }^{*} P<0.05,{ }^{* *} P<0.01,{ }^{* * * *} P<0.001$.

\section{Results}

Erythrocyte specific volume and erythrocyte count in vivo The results showed that red blood cell count of RSSSLD chickens and SLD chickens was higher than that of normal chickens, but the difference between RSS-SLD chickens and SLD chickens was not significant (Table 3). Hematocrit can indirectly reflect the number and volume of erythrocytes, and we found that RSS-SLD chickens and SLD chickens had more hematocrit than normal chickens, the results were consistent with the red blood cell count (Table 3).

\section{Peripheral blood smears}

Figure 1a-f showed that a large number of erythroblasts at various stages in the peripheral blood of RSS-SLD chickens, and some cells were deformed with seriously damaged cell membranes. Erythroblasts were also present in SLD chickens, but this was not obvious comparison with RSS-SLD chickens. Furthermore, comparison of the RSS-SLD chickens with SLD chickens and SLD chickens with normal chickens revealed that the proportion of the erythroblasts (\%) in RSS-SLD chickens and SLD chickens was significantly increased, indicating that the differentiation and maturation of red blood cells was impaired in RSS-SLD chickens and SLD chickens (Fig. 1g).

\section{Bone marrow tissue section results}

Paraffin sections of bone marrow showed that the ratio of yellow fat of RSS-SLD chickens was significantly higher compared with SLD chickens, and that of SLD chickens was significantly higher compared with normal chickens, indicating the bone marrow of SLD chickens was failure and this symptom was more serious in RSSSLD chickens (Fig. 2a-i). Besides, Fig. 2c, f and i showed that large and small cracks appeared in the cartilage of both chicken types, but the symptoms were more serious in RSS-SLD chickens. This indicates that different

Table 3 Determination of erythrocyte specific volume (PCV) and erythrocyte number

\begin{tabular}{llll}
\hline Groups & Number & PCV, $\%$ & Blood red cell count, $\times 10^{9} / \mathbf{m L}$ \\
\hline RSS-SLD & 6 & $46.708 \pm 0.005^{\mathrm{a}}$ & $1.130 \pm 0.018^{\mathrm{a}}$ \\
SLD & 6 & $46.488 \pm 0.025^{\mathrm{a}}$ & $1.028 \pm 0.099^{\mathrm{a}}$ \\
Normal & 6 & $42.997 \pm 0.007^{\mathrm{b}}$ & $0.995 \pm 0.067^{\mathrm{b}}$ \\
\hline
\end{tabular}

a, bwithin a column, values not sharing a common superscript are significantly different degrees of chondral osteoporosis were observed in RSSSLD chickens and SLD chickens, and that bone density was severely reduced. This might be the cause of the skeleton dysplasia, short stature, and easy to appear slope foot in SLD chickens.

\section{BCL6 content and hemoglobin expression in peripheral blood HSCs}

Preliminary work using microarray expression profiling showed that BCL6 expression was upregulated in all dwarf strains. In present study, we speculated that $B C L 6$ was related to the insufficient hematopoietic capacity of RSSSLD chickens and SLD chickens. Therefore, we first explored the functionality of $B C L 6$, and then measured the expression levels of hemoglobin $\alpha_{1}$ and hemoglobin $\beta$. The results showed that the BCL6 expression of RSS-SLD chickens was significantly increased compared with SLD chickens, and that of SLD chickens was significantly increased compared with normal chickens (Fig. 3a). At the same time, we also detected the content of BCL6 via ELISA and confirmed that it was significantly higher in RSS-SLD chickens and SLD chickens (Fig. 3b). Moreover, the hemoglobin $\alpha_{1}$ and hemoglobin $\beta$ expression of RSSSLD chickens were significantly reduced compared to SLD chickens, and that of SLD chickens were significantly reduced compared to normal chickens (Fig. 3c, d).

\section{Determination of various HGFs in plasma by ELISA}

HGFs play an important role in hematopoietic function. To reflect the hematopoietic function of RSS-SLD chickens, SLD chickens and normal chickens, we used ELISA to detect the levels of various HGFs in plasma. The concentrations of EPO, SCF, TPO, and IL-3 of RSS-SLD chickens were all significantly reduced compared to SLD chickens, and that of SLD chickens were all significantly reduced compared to normal chickens (Fig. 4a-d). These HGFs promote the differentiation and development of HSCs, and thus our results indicated that the differentiation and development of HSCs were inhibited in RSSSLD chickens and SLD chickens.

\section{Detection of mitochondrial activity of HSCs in RSS-SLD chickens, SLD chickens and normal chickens}

Mitochondria provide energy for HSCs and maintain the hematopoietic microenvironment. Therefore, HSCs were extracted from the peripheral blood of the chickens to detect mitochondrial function. ROS production was significantly increased, and the ATP concentration along with $\Delta \Psi \mathrm{m}$ was significantly reduced in RSS-SLD chickens' HSCs compared with SLD chickens (Fig. 5a-c). The same results were also observed in SLD chickens' HSCs compared with normal chickens, indicating that impaired mitochondrial function of HSCs in RSS-SLD chickens and 

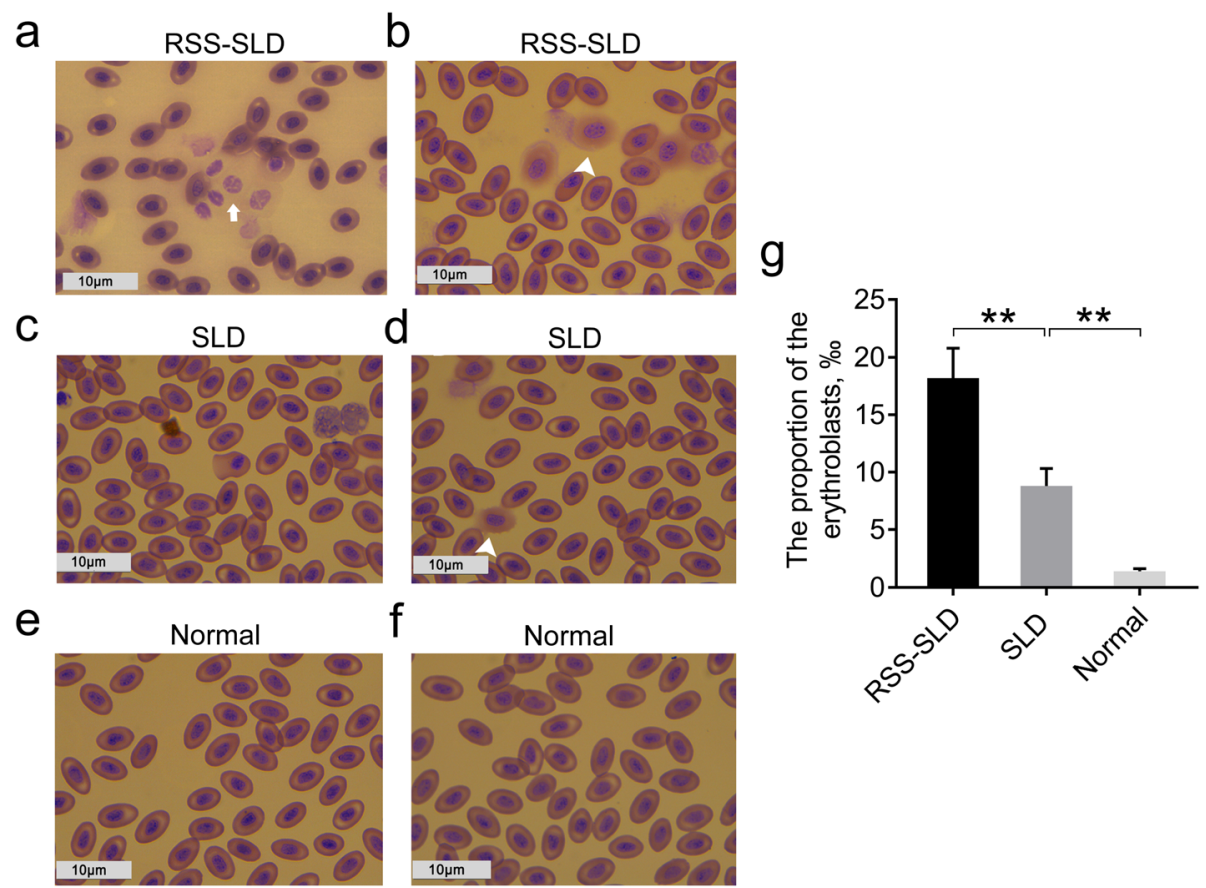

Fig. 1 Results of peripheral blood smears in 140-day-old RSS-SLD chickens, SLD chickens and normal chickens. a and $\mathbf{b}$ are the peripheral blood smears of SLD-RSS chickens; bar $10 \mu \mathrm{m} ; \mathbf{c}$ and $\mathbf{d}$ are the peripheral blood smears of RSS chickens; bar $10 \mu \mathrm{m}$; e and $\mathbf{f}$ are the peripheral blood smears of normal chickens; bar $10 \mu \mathrm{m} ; \mathbf{g}$ The statistical of the proportion of the erythroblasts in the peripheral blood Smears of RSS-SLD, SLD chickens and normal chickens. Data are expressed as means \pm SEM, ${ }^{* *} P<0.01$

SLD chickens might affect the differentiation and development of HSCs in these chickens (Fig. 5a-c).

Effects of overexpression of the BCL6 on two subunits of hemoglobin in HSCs

Next, we studied the effects of the BCL6 on chicken HSCs in vitro (Fig. 6a). The expression of the BCL6 was significantly increased, which was consistent with the ELISA results, indicating successful overexpression of the BCL6 in HSCs (Fig. 6b, c). The expression of both subunits of hemoglobin (hemoglobin $\alpha_{1}$, hemoglobin $\beta$ ) were significantly decreased after BCL6 overexpression, which was consistent with the in vivo results (Fig. 6d, e).
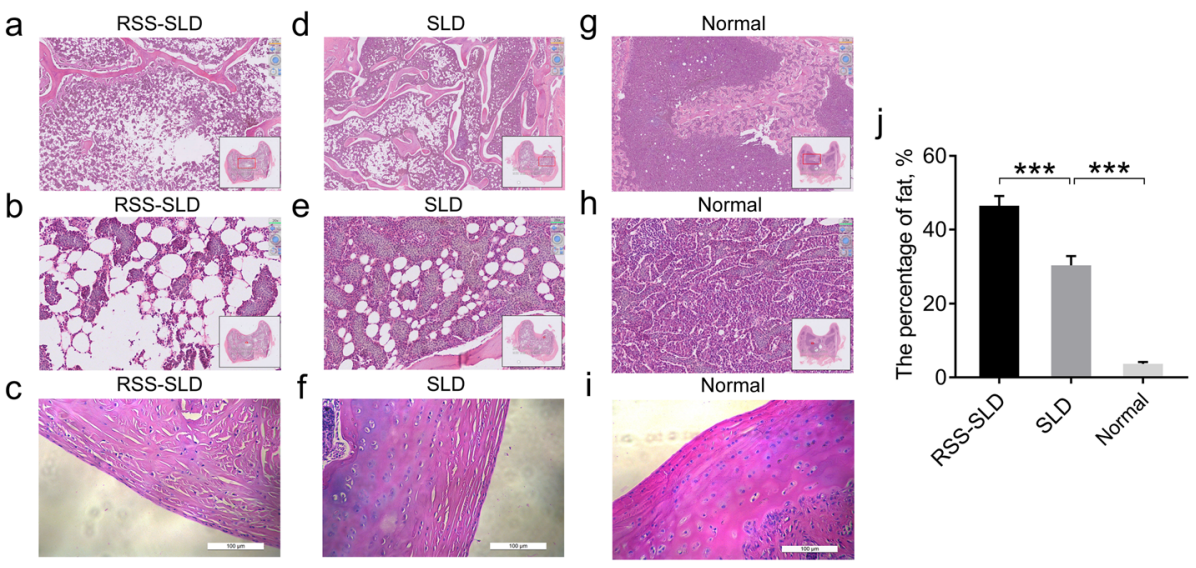

Fig. 2 The bone marrow slices of 140-day-old RSS-SLD chickens, SLD chickens and normal chickens. $\mathbf{a}$ and $\mathbf{b}$ are the bone marrow sections of RSS-SLD chickens; $\mathbf{d}$ and $\mathbf{e}$ are the bone marrow sections of SLD chickens; $\mathbf{g}$ and $\mathbf{h}$ are the bone marrow sections of SLD chickens; $\mathbf{c}, \mathbf{f}$ and $\mathbf{i}$ are the cartilage sections of RSS-SLD, SLD chickens and normal chickens respectively; bar $100 \mu \mathrm{m}$. $\mathbf{j}$ The percentage of fat in volume (\%) of RSS-SLD, SLD chickens and normal chickens. Data are expressed as means \pm SEM, ${ }^{* * *} P<0.001$ 

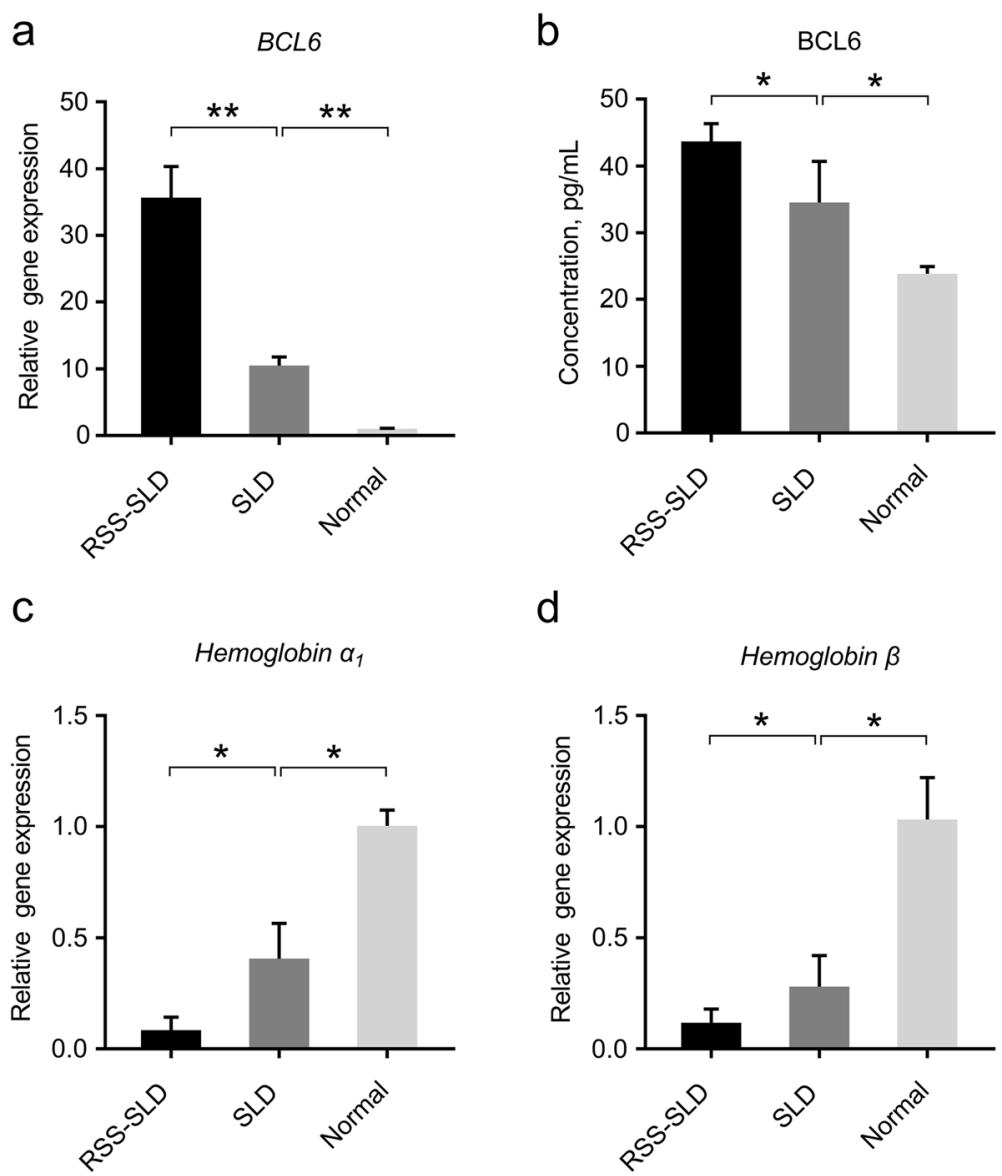

Fig. 3 Relative expression levels of various genes in peripheral blood HSCs of 140-day-old RSS-SLD chickens, SLD chickens and normal chickens. a The HSCs were extracted from peripheral blood and the expression of BCL6 was determined by qRT-PCR. $\mathbf{b} B C L 6$ expression in peripheral blood plasma was determined by ELISA. $\mathbf{c}$ Expression of hemoglobin $a_{1}$ in peripheral blood HSCs. $\mathbf{d}$ Expression of hemoglobin $\beta$ in peripheral blood HSCs. Data are expressed as means \pm SEM, ${ }^{*} P<0.05,{ }^{*} P<0.01$

Effects of overexpression of the BCL6 on HGFs secretion by bone marrow MSCs

Bone marrow MSCs not only provide support for HSCs, but also secrete HGFs to promote the differentiation of HSCs. Therefore, we overexpressed the BCL6 in primary bone marrow MSCs. The bone marrow MSCs phenotype was identified by qRT-PCR verification, and $C D 29$, $C D 44, C D 90$, and $C D 71$ were positive, while CD31, $C D 34$, and $C D 45$ were negative. We also detected the secretion of various HGFs by ELISA (Fig. 7a). The concentrations of EPO, SCF, IL-3, and TPO were all significantly reduced after $B C L 6$ overexpression, indicating that the ability of bone marrow MSCs to secrete cytokines was inhibited (Fig. 7b-e).

\section{Effects of overexpression of the BCL6 on HSCs proliferation and apoptosis}

Next, we studied the effects of $B C L 6$ on HSCs proliferation and apoptosis. The overexpression of the BCL6 promoted the proliferation of HSCs, as detected by CCK8 (Fig. 8a).
Flow cytometry also showed that the percentage of HSCs in the $G_{0} / G_{1}$ phase significantly decreased after $B C L 6$ overexpression (Fig. 8b). However, the percentage of HSCs in the $\mathrm{S}$ phase (DNA synthesis) significantly increased (Fig. $8 b)$. Furthermore, the percentage of HSCs in the $G_{2} / M$ phase did not significantly alter after $B C L 6$ overexpression (Fig. 8b). The $G_{2}$ phase is the late stage of DNA synthesis; cell division begins in the $M$ phase. This indicates that the overexpression of the BCL6 promoted the proliferation of HSCs. Furthermore, we explored the effects of the BCL6 on HSCs apoptosis and found that the proportion of HSCs in the $\mathrm{Q}_{1}$ phase (representing the percentage of living cells) was significantly increased after BCL6 overexpression. Meanwhile, the proportion of HSCs in the $\mathrm{Q}_{2}$ phase (representing early apoptotic cells) was significantly reduced, and that in the $\mathrm{Q}_{3}$ phase (representing late apoptotic cells) did not significantly change after BCL6 overexpression (Fig. 8c). These results demonstrated that overexpression of the $B C L 6$ inhibited the apoptosis of HSCs. 
a

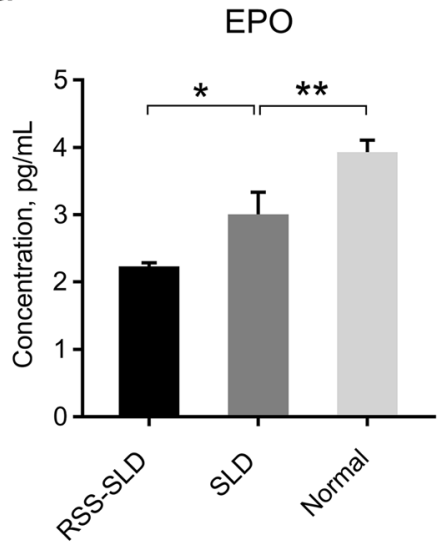

C

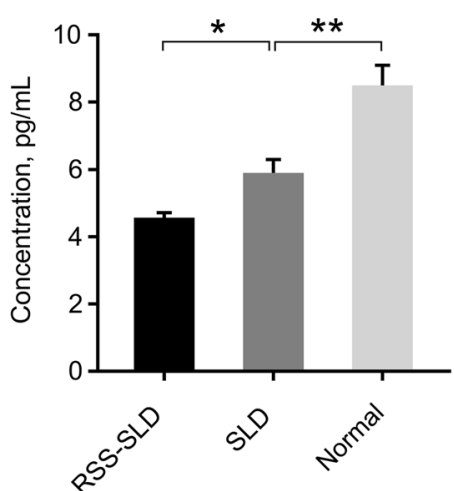

b

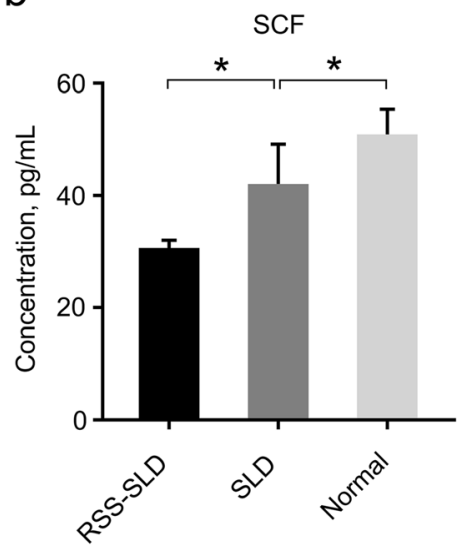

d

IL-3

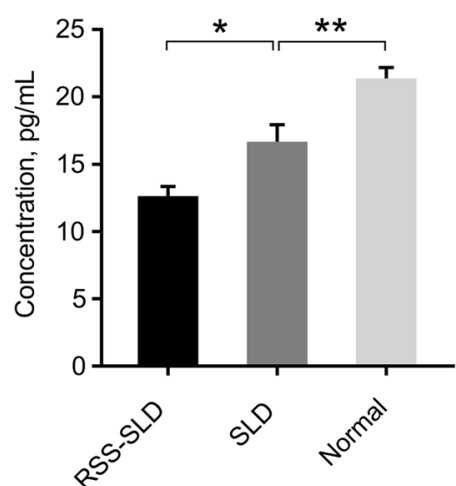

Fig. 4 Determination of various HGFs of 140-day-old RSS-SLD chickens, SLD chickens and normal chickens in plasma by ELISA. a The EPO content in plasma was detected by ELISA. b The SCF content in plasma was detected by ELISA. c The TPO content in plasma was detected by ELISA. $\mathbf{d}$ The IL-3 content in plasma was detected by ELISA. Data are expressed as means \pm SEM, ${ }^{*} P<0.05,{ }^{* *} P<0.01$

a

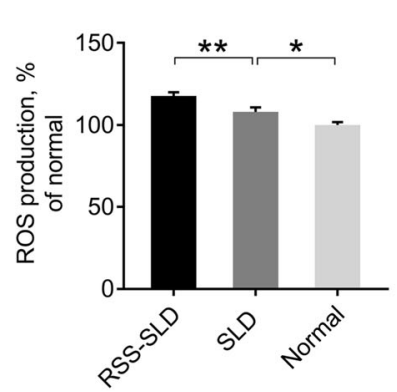

b

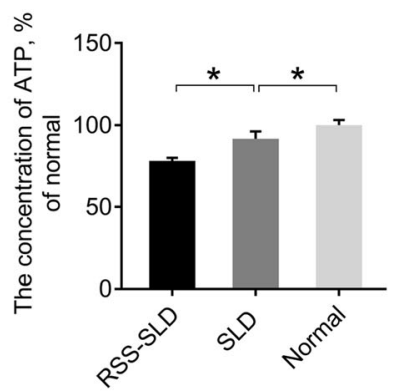

C

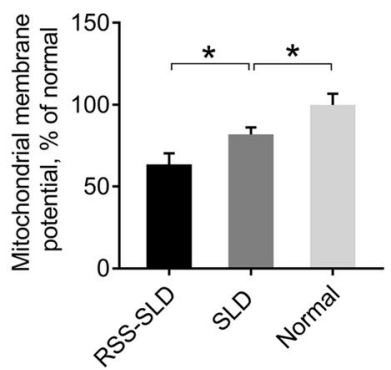

Fig. 5 Detection of mitochondrial function of peripheral blood HSCs in 140-day-old RSS-SLD chickens, SLD chickens and normal chickens. a Detection of ROS levels of peripheral blood HSCs in RSS-SLD chickens, SLD chickens and normal chickens. $\mathbf{b}$ Detection of ATP levels of peripheral blood HSCS RSS-SLD chickens, SLD chickens and normal chickens. c Detection of the $\Delta \psi m$ of peripheral blood HSCs RSS-SLD chickens, SLD chickens and normal chickens. Data are expressed as means $\pm \mathrm{SEM},{ }^{*} P<0.05,{ }^{* *} P<0.01$ 


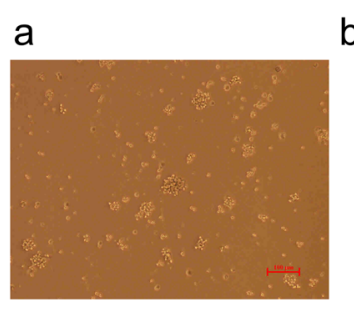

$b$

d

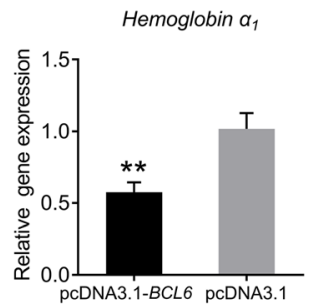

C

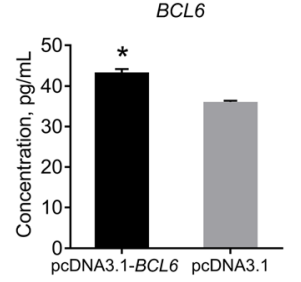

e

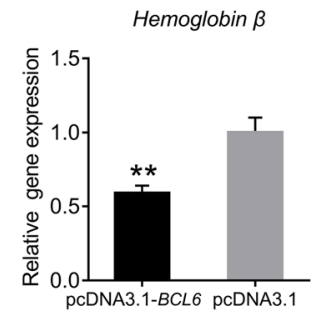

Fig. 6 Morphology of HSCs and the overexpression efficiency of the BCL6. a Morphology of the HSCs; bar $100 \mu$ m. $\mathbf{b}$ Efficiency of the pcDNA3.1$B C L 6$ vector overexpression. c BCL6 expression in HSCs was determined by ELISA. $\mathbf{d}$ Expression of hemoglobin $\mathrm{a}_{1}$. e Expression of hemoglobin $\beta$. Data are expressed as means \pm SEM, ${ }^{*} P<0.05,{ }^{* *} P<0.01$

\section{Effects of overexpression of the $B C L 6$ on mitochondrial}

\section{function of HSCs}

Finally, we detected various mitochondrial function indexes after overexpression of the pcDNA3.1-BCL6 vector in HSCs. ROS production was significantly reduced, and the concentration of ATP along with $\triangle \Psi \mathrm{m}$ was significantly increased after $B C L 6$ overexpression, indicating that the overexpression of the $B C L 6$ promoted mitochondrial function in HSCs (Fig. 9a-c).

\section{Discussion}

RSS in chickens was first described in 1977, the cause of RSS chicken has not been clarified. At present, the research on the causes of RSS have mainly focused on pathogen infection, feeding management, and nutrition levels [10]. In a previous study on RSS-SLD chickens, we observed no inflammatory response or bacterial infection, no reticulum endothelial hyperplasia, subtype J avian leukemia, or Marek's disease [9]. In this study, we

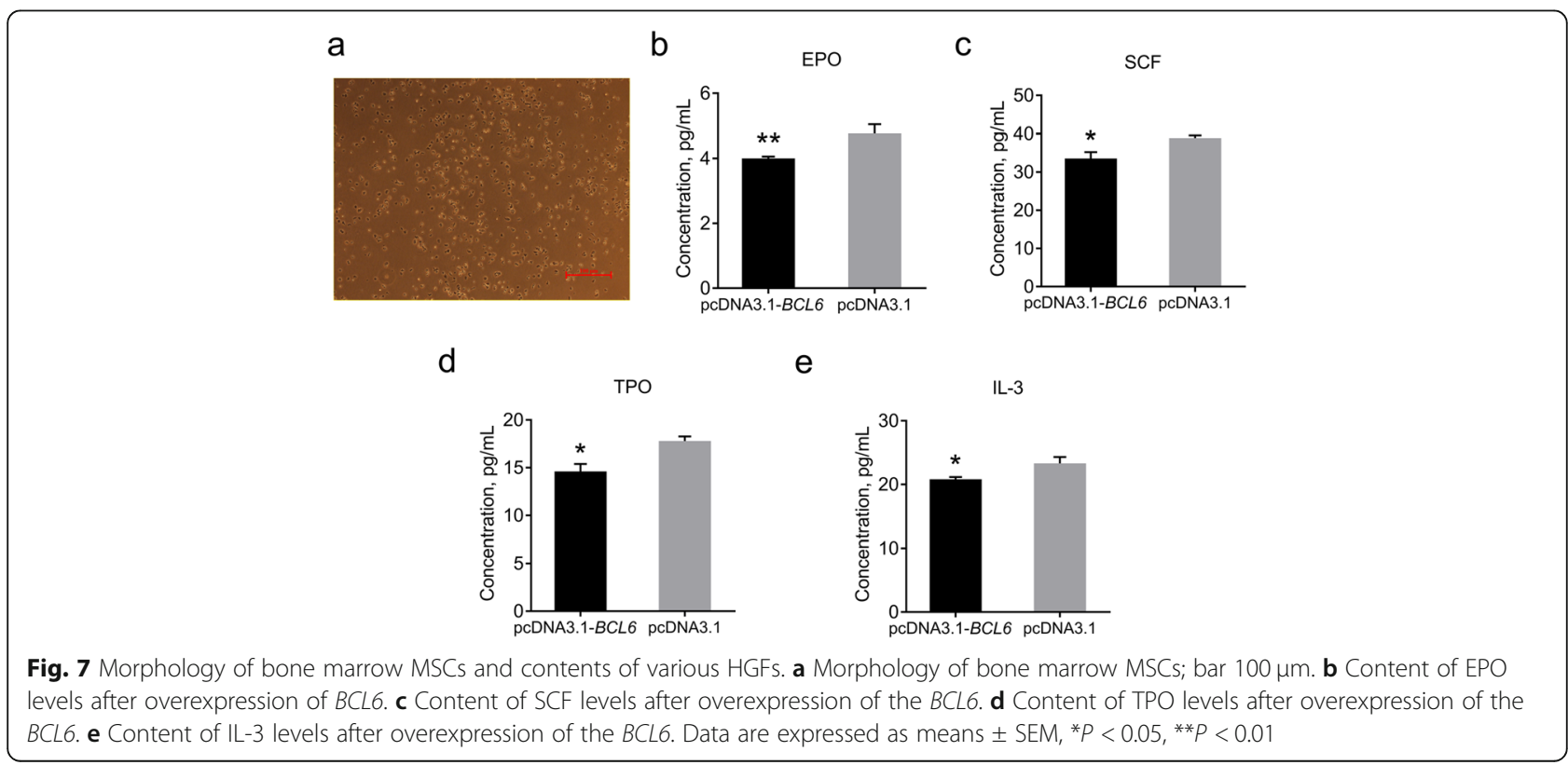




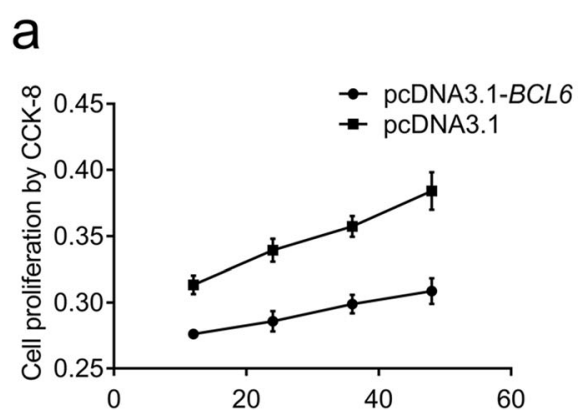

C

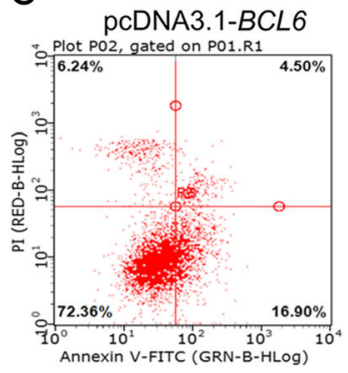

b
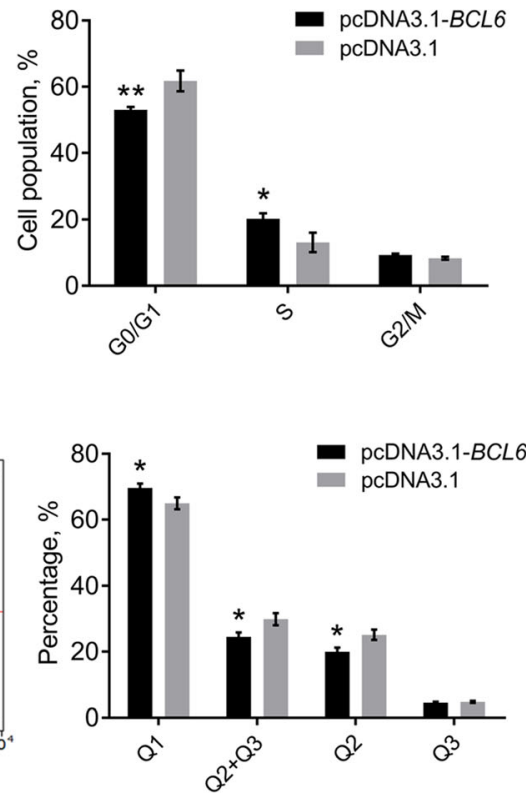

Fig. 8 Effects of overexpression of the BCL6 on HSCs proliferation and apoptosis. a Cell proliferation was detected by CCK8 after overexpression of the BCL6 in HSCs. $\mathbf{b}$ The cell cycle was detected by flow cytometry after overexpression of the BCL6 in HSCs. c Cell apoptosis was detected by flow cytometry after overexpression of the BCL6 in HSCs. Data are expressed as means \pm SEM, ${ }^{*} P<0.05,{ }^{* *} P<0.01$

studied the relationship between the hematopoietic disorder caused by the abnormal expression of BCL6 and RSS in SLD chickens.

The red bone marrow of RSS-SLD chickens changed to yellow bone marrow prematurely, which made the bone marrow enter a pathological state. In the early stages of life, the bone is filled with red bone marrow, which is actively involved in hematopoietic function. Red bone marrow is the body's hematopoietic organ, which can produce red blood cells, platelets, granulocytes, and other blood cells. In addition to the powerful hematopoietic function, red bone marrow has defense, immunity, wound repair, and other functions. Yellow bone marrow is mainly composed of fat, and hematopoiesis ability is weak. The transition from red to yellow bone marrow also signifies aging [12]. Therefore, the change from red to yellow bone marrow in RSS-SLD chickens indicates that the MSCs of these chickens differentiate more readily into fat, and that these chickens may show premature aging.

Accelerated aging of the body leads to significantly decreased function of the immune system (including $\mathrm{B}$ lymphocytes and $\mathrm{T}$ lymphocytes), gradual decreases in the number and quality of bone marrow cells and HSCs [12], and anemia [13]. It is also accompanied by a decrease in bone formation, lower bone density, and an increase in yellow fat in bone marrow. This is consistent with the symptoms of RSS-SLD chickens such as a pale comb, anemia due to insufficient hematopoiesis, stunting, low immunity, and lameness. Bone marrow

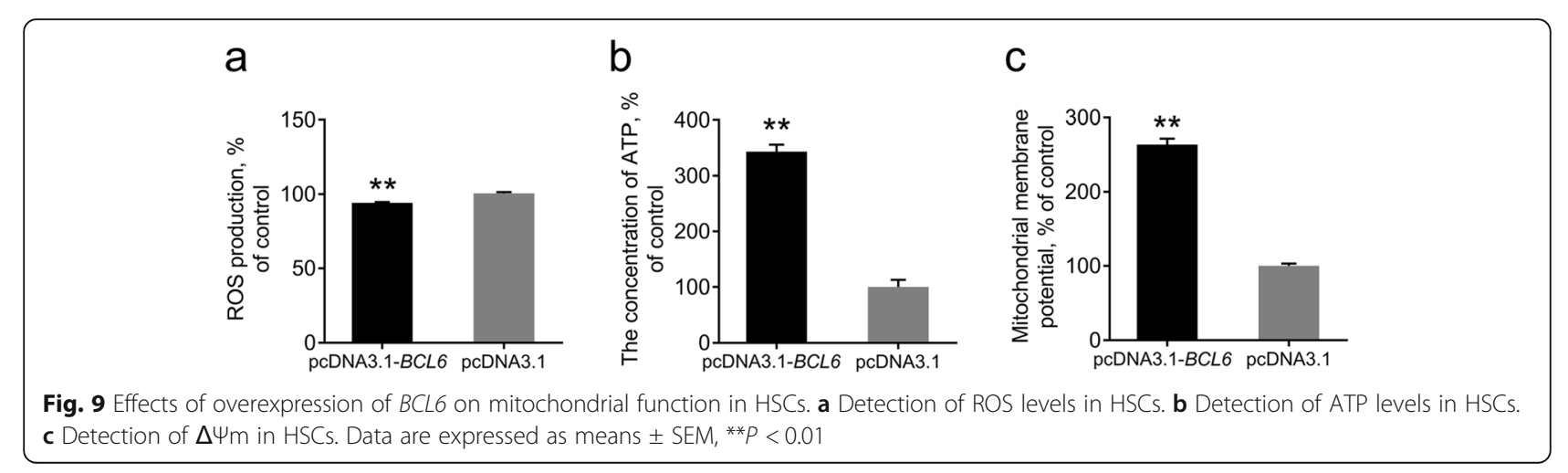


exhaustion leads to the continuous loss of HSCs from the bone marrow niche, resulting in an increase in the number of red blood cells in vitro, which is also a reason for the increase in the number of red blood cells in the peripheral blood of RSS-SLD chickens. According to the results of peripheral blood smears, RSS-SLD chickens had juvenile red blood cells at various stages. Immature red blood cells, including early, middle, and late juvenile cells, are round to oval in shape, with basophilic cytoplasm and light pigmentation, and the nuclei are round to oval in shape with loose chromatin. As the cells mature, the density of chromatin gradually increases, and the cells shrink and become more oval. The abnormalities of red blood cells are mainly reflected as changes in size, number, color, shape, and nature, and such changes can reflect the anemia type, anemia degree, and bone marrow condition of the body [14]. We found that the peripheral blood of RSS-SLD chickens had a large number of young red blood cells at various stages. Moreover, some of the cells were malformed with seriously damaged membranes and low levels of hemoglobin. These results indicate that the hematopoietic function of the RSS-SLD chickens was seriously hindered, but do not reveal the specific mechanism involved.

To further investigate this issue, we examined the function of mitochondria, which affect the differentiation and development of HSCs. ROS levels were significantly higher in the HSCs of RSS-SLD chickens and SLD chickens. ROS are key chemicals in cells, which at controlled concentrations act as second messengers to mediate cellular responses to various endogenous and exogenous signals [15]. However, at high concentrations, they cause redox imbalance and oxidative stress [16]. An increase in ROS can also induce DNA damage. It also affects the copy number of mitochondrial DNA replications and leads to the decline of mitochondrial function [17]. Moreover, the damage of mitochondria will further promote the production of ROS. It is a vicious cycle. Increasing evidence suggests that oxidative stress, particularly ROS levels, affects the development, migration, and self-renewal of stem cells and the state of their cell cycle [18]. ATP levels were significantly lower in RSS-SLD chickens and SLD chickens, possibly due to the damaged mitochondrial structure caused by high ROS levels. A drop in ATP lowers the energy supply to the body, affecting muscle development and HSCs differentiation. This may be why RSS-SLD chickens are mostly listless. The main function of $\Delta \Psi \mathrm{m}$ is to drive the synthesis of ATP through oxidative phosphorylation (OXPHOS). Therefore, the decrease in $\Delta \Psi \mathrm{m}$ in RSSSLD chickens will also affect the synthesis of ATP [19].

Moreover, various hematopoietic cytokines play an important role in the differentiation of HSCs into various daughter cells. They can regulate the survival and proliferation of HSCs and stimulate the differentiation, maturation, and release of HSCs into blood cells of different lines. The decrease in hematopoietic cytokines in RSS-SLD chickens resulted in the differentiation and maturation of stem cells. This is a reason for the decrease in hemoglobin $\alpha_{1}$ and hemoglobin $\beta$ expression in red blood cells.

To further confirm our previous conjecture on the mechanism of low immunity and slow growth in RSSSLD chickens and SLD chickens, we extracted bone marrow HSCs from chickens for cellular verification. The BCL6 gene is an oncogene involved in B-cell lymphoma, which can drive malignant phenotypes by inhibiting DNA damage checkpoints and blocking Bcell terminal differentiation [20]. Normally, most HSCs are in a resting state. Only a small number can be used to maintain the hematopoietic balance by proliferating and differentiating. However, when hematopoietic cells are depleted or damaged, or under the action of an HSC mobilization agent or some cytokines, HSCs will significantly proliferate and more of them will enter the cell cycle to maintain hematopoiesis of the body [21, 22]. The overexpression of BCL6 in cells obviously upset the physiological balance of HSCs. In addition, a large number of hematopoietic cells in RSS-SLD chickens were damaged and depleted, and HSCs were in a pathological state. The infinite proliferation of HSCs inhibits their maturation. These results indicate that the high expression of BCL6 leads to excessive differentiation of MSCs into fat, which is one of the reasons for the increase in yellow bone marrow in RSS-SLD chicken bone marrow.

Overexpression of the BCL6 inhibits chemotherapyinduced ROS production and apoptosis in B-cell lymphoma cells [23]. On the contrary, knockdown of the BCL6 increases hypoxia-induced oxidative stress in cardiomyocytes [24]; our overexpression results are consistent with these findings. Excessive ROS levels can lead to cell senescence and death, while low levels are critical for maintaining the microenvironment of the stem cell pool. $\mathrm{H}_{2} \mathrm{O}_{2}$ in ROS plays an important role in the control of proliferation, differentiation, migration, and activation of signaling pathways [25]. Proto-oncogene BCL6 belongs to the anti-apoptotic family, and BCL6 acts as a transcriptional inhibitor. The target genes regulated by BCL6 are mainly related to cell activation, differentiation, and proliferation, and BCL6 can inhibit cell apoptosis by inhibiting transcription. Mitochondrial dysfunction and ROS accumulation can also promote apoptosis of cancer cells, and lead to the expression of the cell cycle inhibitor $\mathrm{p} 53$, cell cycle arrest in the $\mathrm{G}_{2} / \mathrm{M}$ phase, DNA fragmentation, and apoptosis induction [26]. Chemotherapy drugs such as cyclophosphamide can increase lipid peroxidation and apoptosis induced by ROS in sarcoma- 180 
tumor tissues [27]. In our study, the overexpression of $B C L 6$ decreased the ROS content of HSCs, which promoted the proliferation of HSCs and inhibited their apoptosis.

However, the results of mitochondrial function in vivo are contrary to those in vitro. There is evidence demonstrated that the cells with high $\Delta \Psi \mathrm{m}$ were prone to continue to divide and form tumors, while low $\Delta \Psi \mathrm{m}$ is conducive to the differentiation of embryonic stem cell transplantation mouse stem cells in vivo [28]. In our experiments, $\Delta \Psi \mathrm{m}$ increased after $B C L 6$ overexpression in vitro, confirming the previous finding that overexpression of $B C L 6$ can lead to the oncogenic function of HSCs. On the contrary, the decrease of $\triangle \Psi \mathrm{m}$ in RSS-SLD chicken may indicate $B C L 6$ has a negative effect on mitochondrial function under normal physiological conditions in vivo. Furthermore, the increase in $\Delta \Psi \mathrm{m}$ generally promotes the increase in ATP. In the process of promoting cell proliferation, a large amount of ATP is also produced. Consistently, our results showed that the increase of $\triangle \Psi \mathrm{m}$ and ATP along with the proliferation of HSCs after BCL6 overexpression in vitro. On the other hand, we did not determine whether the ATP came from anaerobic respiration or from normal mitochondrial metabolism. Therefore, we speculated that abnormal proliferation of HSCs after BCL6 overexpression in vitro may be similar to the production of lymphoma, which is powered by anaerobic respiration. This may also be the reason for the discrepant results between in vitro and in vivo experiments, indicating that BCL6 may exert different regulatory mechanisms on mitochondrial function in vivo and in vitro.

There are few reports of the effects of BCL6 on HGFs and the specific mechanism is not clear. In our experiments, overexpression of the BCL6 inhibited the level of HGFs and decreased the expression of hemoglobin $\alpha_{1}$ and hemoglobin $\beta$, similar to the results of RSS-SLD chickens. As a cell suppressor, BCL6 can specifically bind to the promoter DNA of the target gene, thereby inhibiting the transcription of the target gene [29].

\section{Conclusions}

In conclusion, different degrees of mitochondrial dysfunction and HSCs differentiation disorder were observed in RSS-SLD chickens and SLD chickens. Studies at the cellular level found that overexpression of BCL6 would lead to abnormal mitochondrial function, hematopoietic disorder, excessive proliferation, apoptosis inhibition, and a series of cancerous phenomena, indicating that the high expression of BCL6 could inhibit the differentiation and development of HSCs by affecting the mitochondrial function, and might affect the growth and development of chickens.

\section{Abbreviations}

BCL6: B-cell CLL/lymphoma 6; DCF: Dichlorofluorescein; HSCs: Hematopoietic stem cells; HGFs: Hematopoietic growth factors; MSCs: Mesenchymal stem cells; $\Delta \psi \mathrm{m}$ : Mitochondrial membrane potential; nDNA: Nuclear DNA; PCR: Polymerase chain reaction; qRT-PCR: Quantitative real-time PCR; RIPA: Radio immune precipitation assay; RSS: Runting and Stunting Syndrome; ROS: Reactive oxygen species; SLD: Sex-linked dwarf

\section{Acknowledgements \\ Not applicable.}

\section{Authors' contributions}

$\mathrm{HL}$ and $\mathrm{BH}$ contributed equally to this manuscript. $\mathrm{HL}$ participated in the design of the experiment and wrote the manuscript. $\mathrm{BH}$ participated in the design of the experiment and data analyses. SH carried out experiments and analyzed data. WL, DS, MY, ZL, HW, CZ, and DL participated in data collection and interpretation, and helped perform some experiments. MS, $\mathrm{QL}$, and DZ engaged in useful discussion and revised the manuscript. QN and $X Z$ developed the concepts, designed and supervised the study, and wrote the manuscript. The authors read and approved the final manuscript.

\section{Funding}

This work was supported by grants from the Key-Area Research and Development Program of Guangdong Province (Grant No. 2020B020222002), the Guangdong Provincial Promotion Project on Preservation and Utilization of Local Breed of Livestock and Poultry, National Natural Science Foundation of China (Grant No. 31401046), the China Agriculture Research System (CARS-

41-G03), and Guangdong Youth Talent Project.

\section{Availability of data and materials}

All data generated or analyzed during this study are available from the corresponding author on reasonable request.

\section{Ethics approval and consent to participate}

All animal experiments were performed according to the protocols approved by the South China Agriculture University Institutional Animal Care and Use Committee (approval number SCAU\#0017). All animal procedures followed the regulations and guidelines established by this committee and minimized the suffering of animals.

Consent for publication

Not applicable.

\section{Competing interests}

The authors declare that they have no competing interests.

\section{Author details}

${ }^{1}$ Department of Animal Genetics, Breeding and Reproduction, College of Animal Science, South China Agricultural University, Guangzhou 510642, Guangdong Province, China. ${ }^{2}$ Guangdong Provincial Key Lab of AgroAnimal Genomics and Molecular Breeding and Key Lab of Chicken Genetics, Breeding and Reproduction, Ministry of Agriculture, Guangzhou 510642, Guangdong, China. ${ }^{3}$ State Key Laboratory for Conservation and Utilization of Subtropical Agro-bioresources, South China Agricultural University, Guangzhou 510642, Guangdong, China. ${ }^{4}$ Division of Immunology, Virginia-Maryland College of Veterinary Medicine, University of Maryland, College Park, MD, USA.

Received: 1 July 2020 Accepted: 20 December 2020

Published online: 05 February 2021

\section{References}

1. Ohtsuka Y, Arima M, Fujimura L, Li H, Sakamoto A, Okamoto Y, et al. Bcl6 regulates Th2 type cytokine productions by mast cells activated by FcepsilonRI/lgE cross-linking. Mol Immunol. 2005;42:1453-9.

2. Giebel B, Bruns I. Self-renewal versus differentiation in hematopoietic stem and progenitor cells: a focus on asymmetric cell divisions. Curr Stem Cell Res Ther. 2008;3:9-16. 
3. Seita J, Weissman IL. Hematopoietic stem cell: self-renewal versus differentiation. Wiley Interdiscip Rev Syst Biol Med. 2010;2:640-53.

4. Suda T, Arai F, Hirao A. Hematopoietic stem cells and their niche. Trends Immunol. 2005;26:426-33.

5. Juul S, Felderhoff-Mueser U. Epo and other hematopoietic factors. Semin Fetal Neonatal Med. 2007;12:250-8.

6. Chaudhary LR, Spelsberg TC, Riggs BL. Production of various cytokines by normal human osteoblast-like cells in response to interleukin-1 beta and tumor necrosis factor-alpha: lack of regulation by 17 beta-estradiol. Endocrinology. 1992;130:2528-34.

7. Anthony BA, Link DC. Regulation of hematopoietic stem cells by bone marrow stromal cells. Trends Immunol. 2014;35:32-7.

8. Parmar K, Mauch P, Vergilio JA, Sackstein R, Down JD. Distribution of hematopoietic stem cells in the bone marrow according to regional hypoxia. Proc Natl Acad Sci U S A. 2007;104:5431-6.

9. Li H, Hu B, Luo Q, Hu S, Luo Y, Zhao B, et al. Runting and stunting syndrome is associated with mitochondrial dysfunction in sex-linked dwarf chicken. Front Genet. 2019;10:1337.

10. Kang Kl, El-Gazzar M, Sellers HS, Dorea F, Williams SM, Kim T, et al. Investigation into the aetiology of runting and stunting syndrome in chickens. Avian Pathol. 2012:41:41-50.

11. Hu B, Hu S, Yang M, Liao Z, Zhang D, Luo Q, et al. Growth hormone receptor gene is essential for chicken mitochondrial function in vivo and in vitro. Int J Mol Sci. 2019;20:1608.

12. Chambers SM, Goodell MA. Hematopoietic stem cell aging: wrinkles in stem cell potential. Stem Cell Rev. 2007;3:201-11.

13. Linton PJ, Dorshkind K. Age-related changes in lymphocyte development and function. Nat Immunol. 2004;5:133-9.

14. Chen Z. Application of blood smear observation in clinical diagnosis of poultry disease. China Poultry. 2013;35.

15. Ray PD, Huang BW, Tsuji Y. Reactive oxygen species (ROS) homeostasis and redox regulation in cellular signaling. Cell Signal. 2012;24:981-90.

16. Valko M, Rhodes CJ, Moncol J, Izakovic M, Mazur M. Free radicals, metals and antioxidants in oxidative stress-induced cancer. Chem Biol Interact. 2006;160:1-40

17. Liu L, Zhao M, Jin X, Ney G, Yang KB, Peng F, et al. Adaptive endoplasmic reticulum stress signalling via IRE1alpha-XBP1 preserves self-renewal of haematopoietic and pre-leukaemic stem cells. Nat Cell Biol. 2019;21:328-37.

18. Ludin A, Gur-Cohen S, Golan K, Kaufmann KB, Itkin T, Medaglia C, et al. Reactive oxygen species regulate hematopoietic stem cell self-renewal, migration and development, as well as their bone marrow microenvironment. Antioxid Redox Signal. 2014;21:1605-19.

19. Logan A, Pell VR, Shaffer KJ, Evans C, Stanley NJ, Robb EL, et al. Assessing the mitochondrial membrane potential in cells and in vivo using targeted click chemistry and mass spectrometry. Cell Metab. 2016;23:379-85.

20. Cardenas MG, Oswald E, Yu W, Xue F, MacKerell AJ, Melnick AM. The expanding role of the BCL6 oncoprotein as a cancer therapeutic target. Clin Cancer Res. 2017;23:885-93.

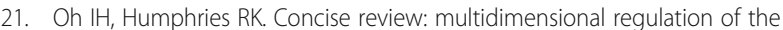
hematopoietic stem cell state. Stem Cells. 2012;30:82-8

22. Watts KL, Adair J, Kiem HP. Hematopoietic stem cell expansion and gene therapy. Cytotherapy. 2011;13:1164-71.

23. Kurosu T, Fukuda T, Miki T, Miura O. BCL6 overexpression prevents increase in reactive oxygen species and inhibits apoptosis induced by chemotherapeutic reagents in B-cell lymphoma cells. Oncogene. 2003:22: 4459-68.

24. Gu Y, Luo M, Li Y, Su Z, Wang Y, Chen X, et al. Bcl6 knockdown aggravates hypoxia injury in cardiomyocytes via the P38 pathway. Cell Biol Int. 2019;43: 108-16.

25. Rhee SG. Cell signaling. H2O2, a necessary evil for cell signaling. Science. 2006:312:1882-3.

26. Wu CL, Huang AC, Yang JS, Liao CL, Lu HF, Chou ST, et al. Benzyl isothiocyanate (BITC) and phenethyl isothiocyanate (PEITC)-mediated generation of reactive oxygen species causes cell cycle arrest and induces apoptosis via activation of caspase-3, mitochondria dysfunction and nitric oxide (NO) in human osteogenic sarcoma U-2 OS cells. J Orthop Res. 2011 29:1199-209.

27. Jana S, Patra K, Sarkar S, Jana J, Mukherjee G, Bhattacharjee S, et al. Antitumorigenic potential of linalool is accompanied by modulation of oxidative stress: an in vivo study in sarcoma-180 solid tumor model. Nutr Cancer. 2014;66:835-48.
28. Schieke SM, Ma M, Cao L, McCoy JJ, Liu C, Hensel NF, et al. Mitochondrial metabolism modulates differentiation and teratoma formation capacity in mouse embryonic stem cells. J Biol Chem. 2008;283:28506-12.

29. Basso K, Dalla-Favera R. Roles of BCL6 in normal and transformed germinal center B cells. Immunol Rev. 2012;247:172-83.
Ready to submit your research? Choose BMC and benefit from:

- fast, convenient online submission

- thorough peer review by experienced researchers in your field

- rapid publication on acceptance

- support for research data, including large and complex data types

- gold Open Access which fosters wider collaboration and increased citations

- maximum visibility for your research: over $100 \mathrm{M}$ website views per year

At BMC, research is always in progress.

Learn more biomedcentral.com/submissions 